\title{
Procedimiento para el procesamiento de información científica en la DPI de la carrera Ingeniería Forestal
}

\author{
Ana Luisa Figueredo Figueredo \\ Rafael Francisco León Aguilar \\ Mariela María Martínez Roselló \\ Universidad de Granma - UDG, Cuba
}

\section{CASE REPORT}

\begin{abstract}
Resumen
Objetivo. Elaborar un procedimiento para el procesamiento de información científica en la Disciplina Principal Integradora de la Carrera Ingeniería Forestal de la Universidad de Granma (Cuba), derivado en tres objetivos específicos: diagnosticar el procesamiento de información científica; diseñar un procedimiento para el procesamiento de información científica y valorar la efectividad del procedimiento en el proceso de formación profesional de pregrado.

Métodos. Se aplicaron como métodos teóricos el histórico tendencial, análisis-síntesis y deducción-inducción; de los métodos empíricos, encuesta, observación científica-participativa y como estadístico, la estadística descriptiva.

Resultados. Los autores aportan un procedimiento para el procesamiento de información científica a través del uso de diferentes fuentes de información, teniendo en cuenta la evidente necesidad de resolver un grupo de limitaciones que impiden la adecuada utilización de las fuentes que tributan a la formación profesional de los Ingenieros Forestales.

Conclusiones. En el estudio se revela que el uso adecuado de cada fuente de información potencia la calidad del proceso de enseñanza - aprendizaje, estableciendo nuevas relaciones en torno al procesamiento de información científica.
\end{abstract}

Palabras-clave

Enseñanza superior; Información científica; Procesamiento de información científica.

Procedure for the processing of scientific information in the DPI of the Forest Engineering programme

\begin{abstract}
Objective. Develop a procedure for the processing of scientific information in to the Disciplina Principal Integradora (DPI) of the Forest Engineering Programme on the University of Granma (Cuba), derivative in specific objectives: diagnose the processing of scientific information; designing a procedure for the processing of scientific information and assess the effectiveness of the procedure in the process of training of undergraduate.

Method. Applied as a theoretical methods, historical trend, analysis - synthesis and deduction - induction; empirical methods, survey, observation scientific - participative and as statistical, descriptive statistics.

Results. The authors provide a procedure for the processing of scientific information through the use of different informational stands, from the obvious need of solving a group of constraints that prevent the proper use of sources that taxed to the vocational training of foresters.

Conclusions. The study reveals that the proper use of each source of information power the quality of the teaching process - learning, establishing new relationships around the processing of scientific information.
\end{abstract}

Keywords

Higher education; Processing of scientific information; Scientific information.

\section{Introducción}

El procesamiento de la información científica en la educación superior no ha sido muy abordado en el ambiente académico, sin embargo, existen un grupo de investigaciones, tanto nacionales como internacionales, que 
demuestran la necesidad de prestar atención a las vías, formas y herramientas empleadas para realizar un tratamiento adecuado de la información disponible.

No se puede hacer referencia en la actualidad a un eficiente desarrollo del proceso docente_educativo, investigativo y extensionista, en el que no esté presente el procesamiento de información científica. A través del empleo eficiente de documentos básicos y complementarios se ponen de manifiesto un grupo de funciones como la transmisión de la información, adquisición de conocimientos esenciales, desarrollo de habilidades intelectuales y prácticas, así como estímulo al proceso de estudio. Atendiendo a lo anteriormente planteadose define entonces como objetivo general: Elaborar un procedimiento para el procesamiento de información científica en la Disciplina Principal Integradora de la Carrera Ingeniería Forestal.

Para comprender el comportamiento del tema se hace necesario precisar un grupo de términos, que permitirán conducir las relaciones lógicas que se establecen en el desarrollo de la investigación. Núñez (2004) plantea que "el concepto de información ha sido tratado ampliamente, desde los puntos de vista de diferentes ciencias, en unas como proceso o acción de informar y en otras, como unidad significante/significado que existe en las fuentes". Cruz y García (1994) definen la información como "la forma social de existencia del conocimiento consolidada en una fuente determinada" (p.24).

Para las investigadoras González, Hernández y Viñas (2001) "procesar información significa analizarla, delimitar en ella los hechos, conceptos, distinguir las posiciones principales del autor, las argumentaciones, sistematizar o reorganizar lógicamente el contenido, resumirlo" (p.48). Estas y otras acciones más allá del establecimiento de los antecedentes del tema.

"El procesamiento de la información es un continuo que va desde un procesamiento superficial, pasando por uno intermedio hasta llegar al más profundo, de carácter semántico, de construcción de significado... La persistencia de la información que almacenamos en nuestra memoria está en función de la profundidad del análisis. En consecuencia, los niveles de análisis más profundos permiten que dicha información sea más elaborada, más fuerte y más perdurable. A mayor grado de análisis semántico, mayor profundidad de procesamiento" (Gómez, 2004, p.289).

Los autores coinciden con este criterio teniendo en cuenta que el procesamiento de información lo tiene incorporado a su vida cotidiana, toda persona con la capacidad de razonar y en dependencia de diferentes factores, internos y/o externos, varía su nivel de profundidad. Es un hecho demostrado que mientras más información se procese, más se perfeccionan las habilidades cognitivas que posee el individuo, así como las vías y formas para llegar al resultado deseado.

"El procesamiento de la información a partir del documento científico es concebido como una red de ideas interconectadas y como una trama de intenciones elaborada o reconstruida por los comunicantes en función de los esquemas de conocimientos compartidos. El procesamiento cognitivo del documento se deriva de un compromiso de negociación entre la información explicita y la información tácita" (Lancaster y Pinto, 2001, p.210).

En la revisión bibliográfica realizada se encontraron elementos sobre algunas de las formas de procesamiento de información científica de forma independiente, pero no se encontró ningún documento que hiciera referencia, de forma exclusiva, al comportamiento de este para la actividad forestal. Es necesario destacar que esta situación se comporta de manera similar para otras áreas del conocimiento, con excepción de las Matemáticas, donde se cuenta con investigaciones relacionadas al desarrollo de habilidades lógicas para la solución de situaciones problémicas.

En las Ciencias Forestales se pueden mencionar las contribuciones de Notario (1999), quien hace referencia a la investigación documental, qué se lee y cómo se lee (técnicas de lectura de documentos científicos), así como elementos esenciales para la elaboración de fichas bibliográficas. Refiere, de forma particular, los principales tipos de documentos necesarios para desarrollar una investigación documental, criterio que se tuvo en cuenta para determinar la propuesta de las principales fuentes de información a consultar para el procesamiento de información científica en la DPI. Revela, además, de manera clara y precisa, elementos medulares para el procesamiento de información en la investigación científica. 
Como principales limitaciones para el desarrollo de la investigación se tienen que: en los documentos consultados se encontró poca información sobre la aplicación de procedimientos para el procesamiento de información científica en la educación superior; en la Disciplina Principal Integradora de la carrera Ingeniería Forestal no se han aplicado instrumentos (encuestas y entrevistas) relacionados al tema, a partir de los cuales se puedan establecer comparaciones entre varios cursos académicos; aún es insuficiente el uso que hacen los estudiantes de las diferentes fuentes de información disponibles para la disciplina, donde por el sistema de evaluación diseñado para las asignaturas que la conforman, se tiene que hacer un exhaustivo uso de habilidades relacionadas al manejo de información científica.

\section{Metodología}

La investigación se desarrolló en tres etapas fundamentales: diagnóstico del estado actual del procesamiento de información científica en la DPI, diseño y valoración del procedimiento, lo cual permitió la aplicación de las diferentes técnicas e instrumentos hasta lograr el diseño definitivo del procedimiento.

La primera etapa comenzó con la aplicación de encuestas (Anexo I) a estudiantes de primero a quinto año de la Carrera Ingeniería Forestal, para determinar los elementos esenciales del estado actual del procesamiento de información científica, sirviendo de base para la elaboración de un procedimiento que pudiera ser aplicado a nivel de carrera.

La muestra se determinó a partir del criterio de Calero (1978), procedimiento planteado para estudios sociales en poblaciones finitas o conocidas. El tipo de muestreo realizado fue aleatorio estratificado para un nivel de significación de un $5 \%(\alpha=0,05)$ y una precisión de 0,05 , con fijación al tamaño del estrato. Los estratos se determinaron teniendo en cuenta los años académicos: 12 estudiantes en primero, 18 en segundo, 17 en tercero, 31 en cuarto y 34 en quinto.

Se revisaron los programas analíticos de cada una de las asignaturas que conforman la DPI para consultar el objetivo formativo, sistema de conocimientos y sistema de habilidades de cada una de ellas. Esto fue esencial para el enfoque de sistema con que fue concebido el procedimiento.

Se clasificaron las fuentes de información atendiendo al criterio de Cruz Paz (1992), donde se incluye, además de las documentales, las personales, institucionales e impersonales (pp.267-272). Para la Carrera Ingeniería Forestal es muy importante el trabajo con las fuentes de información impersonales, pues el objeto de la profesión es el manejo sostenible de los recursos forestales. Esta clasificación fue parte esencial para la posterior evaluación de las fuentes que conforman los diferentes fondos y colecciones.

Para la evaluación de los fondos y colecciones a los que tienen acceso los estudiantes de esta carrera en la DPI se propone tener en cuenta tanto elementos cualitativos como cuantitativos, a partir de un grupo de indicadores en los que se recoge la esencia del nivel de pertinencia de estos. Siguiendo elementos esenciales de los criterios de Tomaél, et al. (2001) y Kirk (2001), los indicadores propuestos para la evaluación son: autoría, actualidad, estado de conservación, uso y accesibilidad.

Los criterios fueron desarrollados a partir de la consulta a profesores de la DPI, especialistas, bibliotecarios e informáticos. Se desarrolló el levantamiento de todas las fuentes de información empleadas en la DPI, tanto las básicas como las complementarias. Dentro de los fondos y colecciones evaluados se encuentran: almacén de préstamo a estudiantes, biblioteca universitaria, plataforma Moodle y ftp, gabinete metodológico del Departamento Ingeniería Forestal, xiloteca, arboretum y colección de rocas. Cada uno de ellos se evaluó de forma independiente, teniendo en cuenta como criterios de evaluación: autoría (excluyendo las fuentes impersonales), actualidad del contenido (solo para las fuentes documentales), estado de conservación, uso y accesibilidad.

Se realizó la revisión de los Trabajos de Curso (2014-2015 y 2015-2016) de todas las asignaturas de la DPI. El propósito fue establecer comparaciones entre los tipos de procesamiento que se evidencian en su elaboración, las fuentes de información y habilidades más empleadas. Posteriormente se determinaron las acciones y operaciones que conformaron el procedimiento: tres acciones con un sistema de operaciones. 
Para la tercera y última etapa, se aplicó una entrevista semi-estandarizada (Anexo 2) a profesores y especialistas, encaminada a la valoración del procedimiento. Se desarrollaron, además, talleres con los estudiantes de la carrera con el objetivo de socializar el procedimiento y someterlo a la valoración de estos. En la primera y última etapa, para el procesamiento de cuestionarios, observación científica, evaluación de fuentes y entrevistas se utilizó el software SPSS (versión 21), lo que posibilitó mayor precisión y cientificidad en la interpretación de los resultados obtenidos.

\section{Resultados y discusión}

A nivel de carrera la vía de acceso a las fuentes de información más empleada es el almacén de préstamo, con marcada diferencia respecto al resto, por lo que se puede inferir que son las fuentes impresas las que tienen un mayor uso. Resulta contradictorio que si la sociedad está inmersa en la llamada Era de la Información y el Conocimiento, donde, como resultado del empleo de las TIC la información se incrementa a un ritmo incontrolable, los estudiantes universitarios tengan identificado como la vía más utilizada, el almacén de préstamo de libros. (Fig. 1).

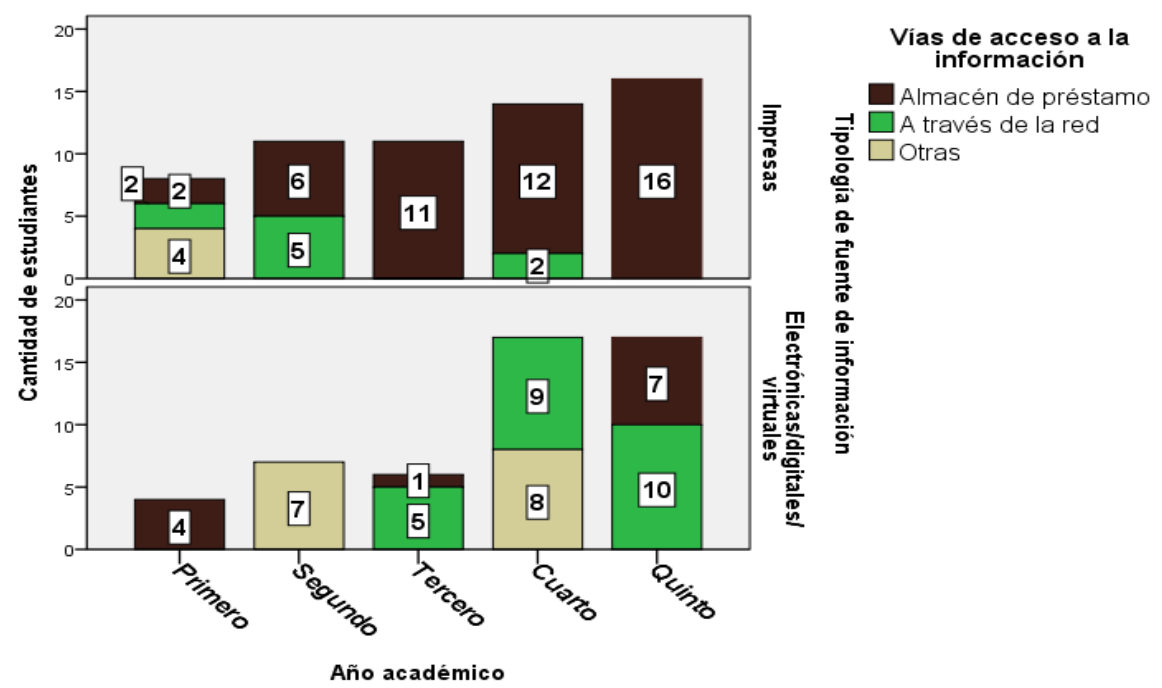

Fig.1. Tipología de fuentes de información y vías de acceso por años académicos Fuente: Elaboración de los autores

En la DPI no es significativa la consulta de que realizan los estudiantes. Se hace necesario trabajar intencionadamente el uso de diferentes fuentes de información en una disciplina cuya evaluación final del mayor número de sus asignaturas es un Trabajo de Curso y en los casos específicos de Metodología de la Investigación y Trabajo de Diploma, tienen que realizar un uso intenso de habilidades relacionadas al manejo de información científica. (Fig. 2). 


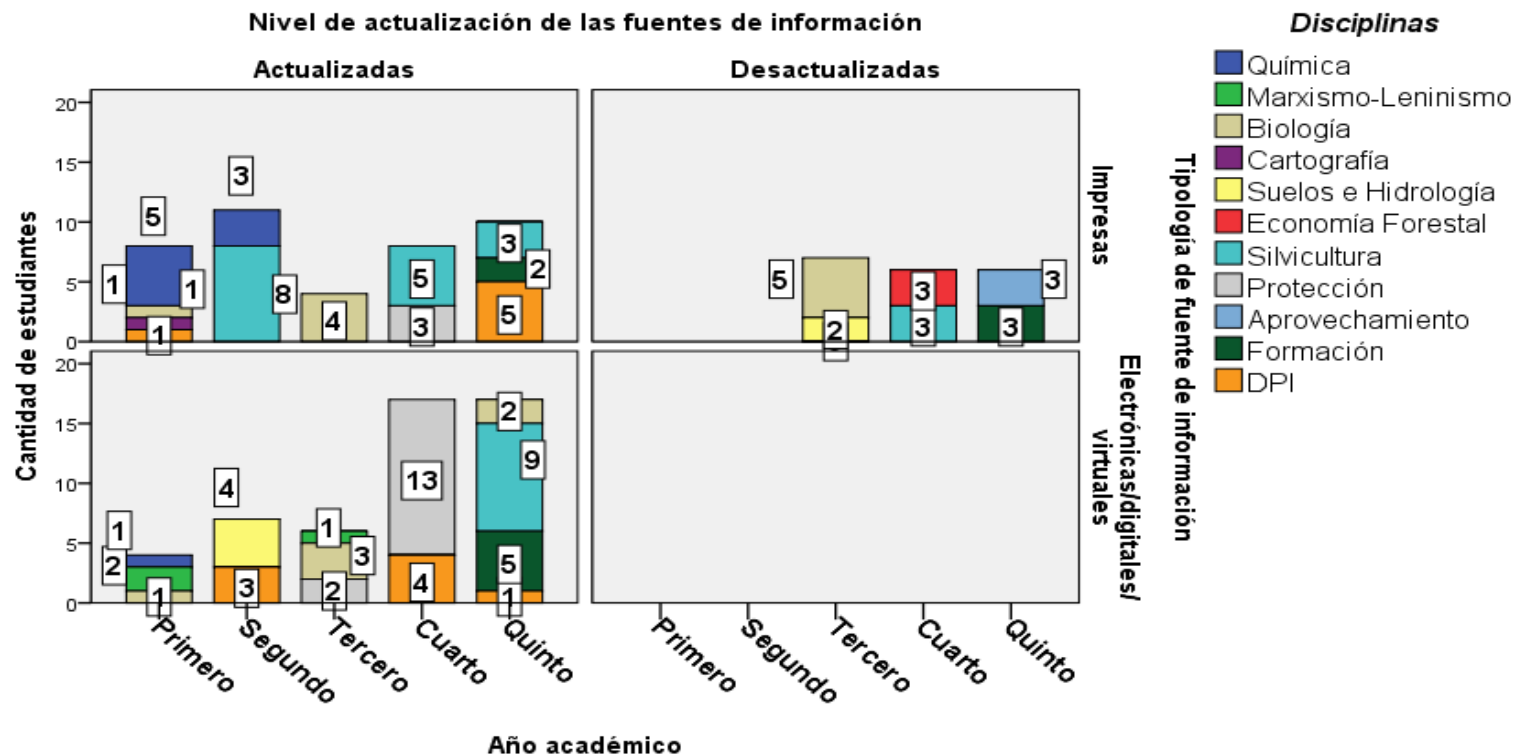

Fig. 2. Tipología de fuentes de información y nivel de actualización por disciplinas Fuente: Elaboración de los autores

Para determinar las fuentes de información más empleadas por los estudiantes, estas se agruparon por soportes (Fig. 3). En la medida que el estudiante transita por los diferentes años se va incrementando la consulta de documentos en soporte electrónico.

Resulta paradójico el hecho de que, existiendo mejores oportunidades en el acceso a fuentes de información en soporte electrónico, continúe siendo el libro impreso el documento que más consultan los estudiantes y que la revista electrónica, que posee una rápida circulación y un alto grado de actualización, se usada por un número reducido de estudiantes. No obstante, también reconocen usar el libro en formato electrónico, cuyo proceso de edición es mucho más rápido que el libro en soporte impreso, por lo tanto, el nivel de actualización de los contenidos es mayor. Aún es insuficiente la consulta de Trabajos de Diploma y guías de estudio.

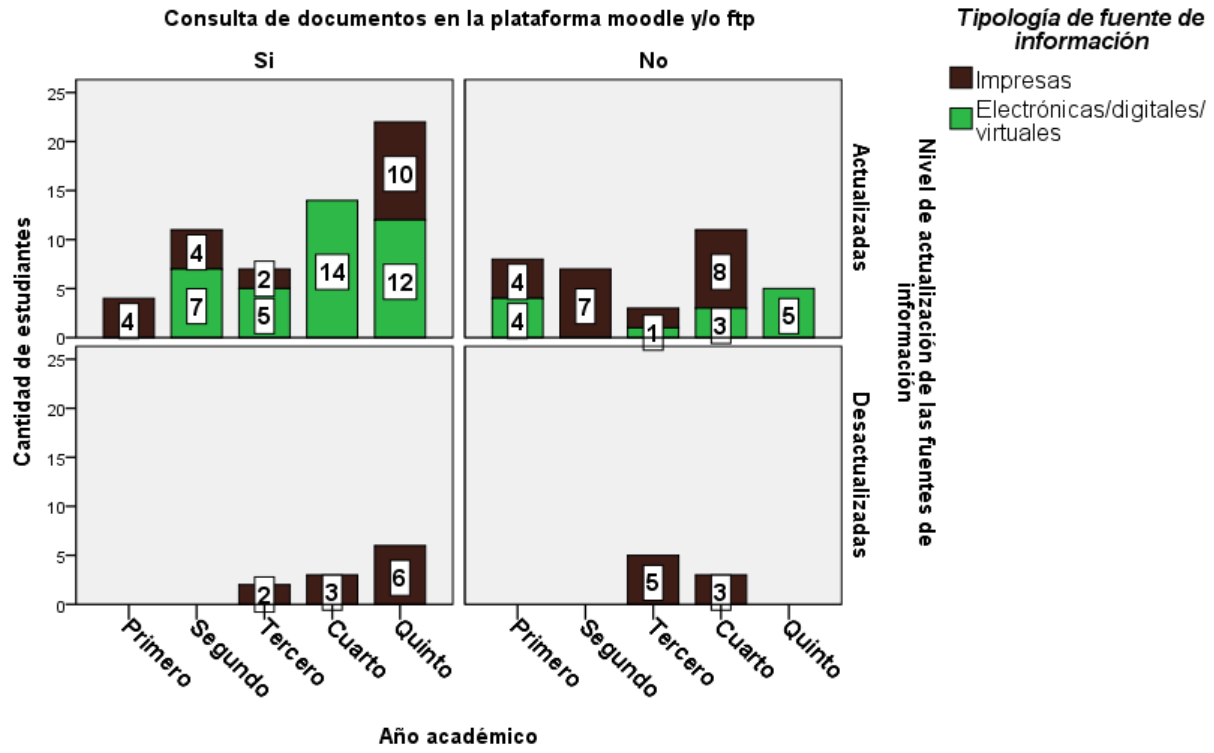

Fig. 3. Consulta de documentos en la Plataforma Moodle y ftp, tipología de fuentes y nivel de actualización por años académicos

Fuente: Elaboración de los autores 
Los diferentes tipos de procesamiento de información se integraron en dos grupos: procesamiento simultáneo (elaborar informes, trabajos de curso, ponencias) y procesamiento secuencial (lectura de estudio, resumen, esquema, mapa conceptual, gráfico, bibliografía, fichar y tomar nota). El criterio asumido para esta clasificación fue el de Ruiz (2004), quien "dentro del procesamiento simultáneo agrupa habilidades como analizar, sintetizar, comparar, abstraer, caracterizar, definir, identificar, clasificar, ordenar, relacionar, interpretar, explicar, observar, memorizar, argumentar, evaluar y dentro del procesamiento secuencial, representar, resumir, fichar, anotar, leer"(p.152).

"Las habilidades de cada disciplina podemos clasificarlas, según su nivel de sistematicidad en: las propias de la ciencia específica; las habilidades lógicas, tanto formal como dialéctica, también llamadas intelectuales o teóricas, las cuales se aplican en cualquier ciencia, tales como inducción-deducción, análisis-síntesis, generalización, abstracción-concreción, clasificación, definición, las de la investigación científica, etc. Además se presentan las habilidades propias del proceso docente en sí mismo, y de autoinstrucción, tales como tomar notas, la realización de resúmenes y de fichas, el desarrollo de informes, la lectura rápida y eficiente, entre otros" (Álvarez, 1999, p.218). Para clasificar las habilidades señaladas por los estudiantes se siguió el criterio del autor anteriormente referenciado.

En este caso, el número de estudiantes que señalan aplicar un procedimiento para el procesamiento de información es bajo con relación a los que no lo hacen (Fig. 4), sin embargo, en los primeros es superior la manifestación de habilidades de autoinstrucción, asociadas al procesamiento de información secuencial. Llama la atención el hecho de que los estudiantes reconocen como una de las habilidades más utilizadas, memorizar, y sin embargo pocos se refieran a representar.

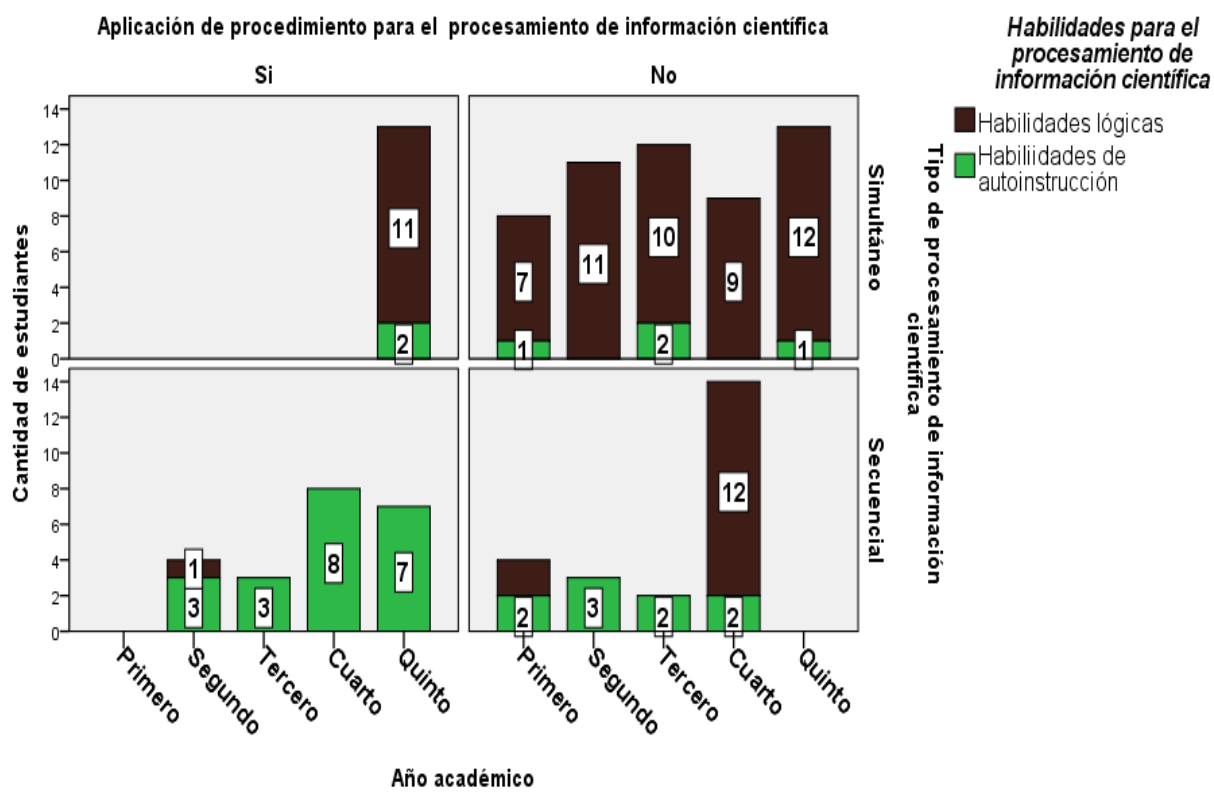

Fig. 4. Habilidades, tipo de procesamiento de información científica y aplicación de procedimiento Fuente: Elaboración de los autores

La mayoría de los estudiantes no perciben que, aunque sea de forma instintiva, de alguna manera ellos aplican algún tipo de procedimiento para el procesamiento de información, aunque este no sea siempre el más efectivo. Se infiere una vez más, la necesidad de proponer un procedimiento que permita al estudiante hacer un uso adecuado de las diferentes fuentes de información.

Para determinar la actualidad de las fuentes de información (Fig. 5) se consultaron los profesores de la DPI, realizándose fuente por fuente. No se tuvieron en cuenta las correspondientes a la Xiloteca, Colección de rocas y Arboretum, pues este criterio no responde al tipo de fuente que los conforman. Atendiendo al nivel de 
actualidad de cada uno de los fondos, se puede plantear que de forma general solo un $6.31 \%$ se encuentra desactualizado, lo que constituye una fortaleza para el trabajo en la disciplina.

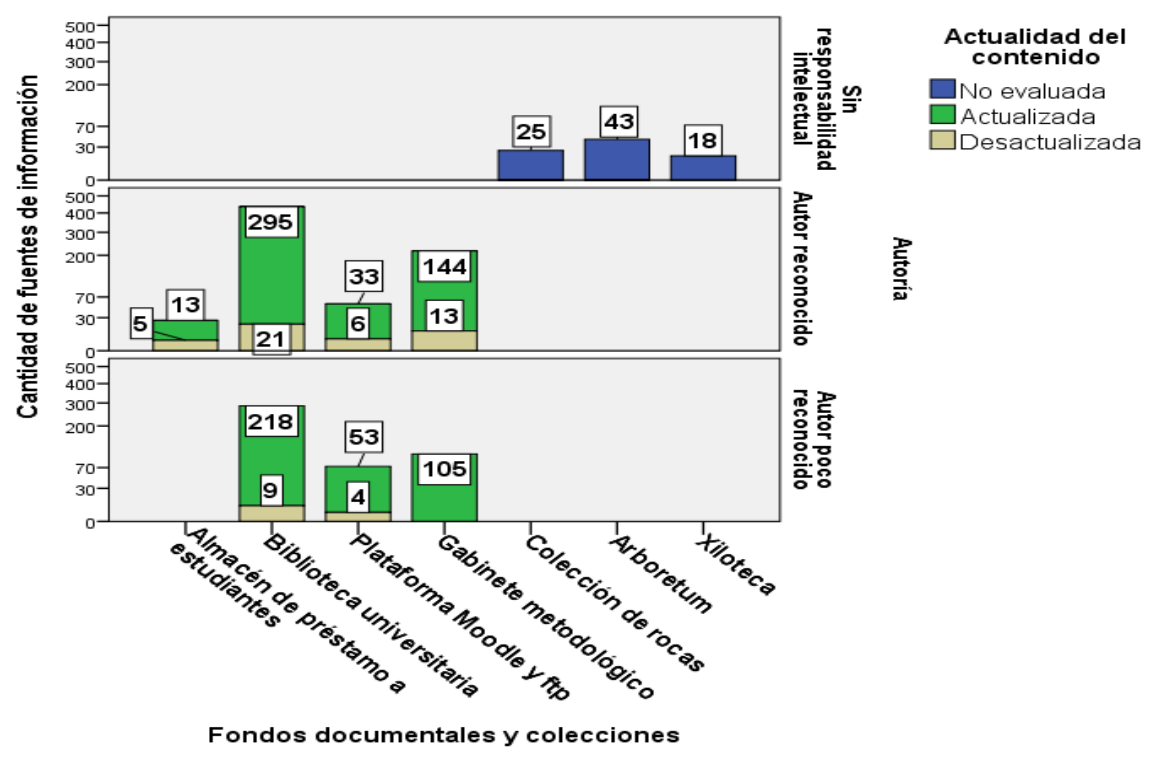

Fig. 5. Actualidad de las fuentes de información Fuente: Elaboración de los autores

Es necesario señalar que, de los documentos desactualizados, el comportamiento de este estado no es para el $100 \%$ del contenido de estos documentos, sino para una parte de ellos, por eso continúan formando parte de los fondos.

En relación, a la accesibilidad a cada una de las fuentes de información, por fondo y colección, solo el 0.59 \% es de difícil acceso. Este se refiere a los títulos del almacén de préstamo a estudiantes, cuya distribución entre los estudiantes es por equipos. El elevado por ciento en la facilidad para acceder a las fuentes se considera una fortaleza, pues esto facilita la recuperación de la información.

En cuanto al estado de conservación, en el almacén de préstamo el dato coincide con el de las fuentes de difícil acceso, pues al ser reducido el número de ejemplares de esos títulos, se incrementa su uso, deteriorándose con mayor facilidad que aquellos para los cuales es mayor la distribución por estudiantes.

El uso se evaluó a partir de la consulta a profesores de la DPI, técnica en gestión que atiende el almacén de préstamo a estudiantes, bibliotecarias y profesores responsables de las colecciones del departamento. No se pudo evaluar el uso de los documentos electrónicos, pues no se contó con ninguna herramienta informática que hiciera posible esta evaluación. Se considera preocupante que el $65.48 \%$ se corresponda con el uso pasivo de las fuentes y solo el $34.52 \%$ al activo (Fig.6). 


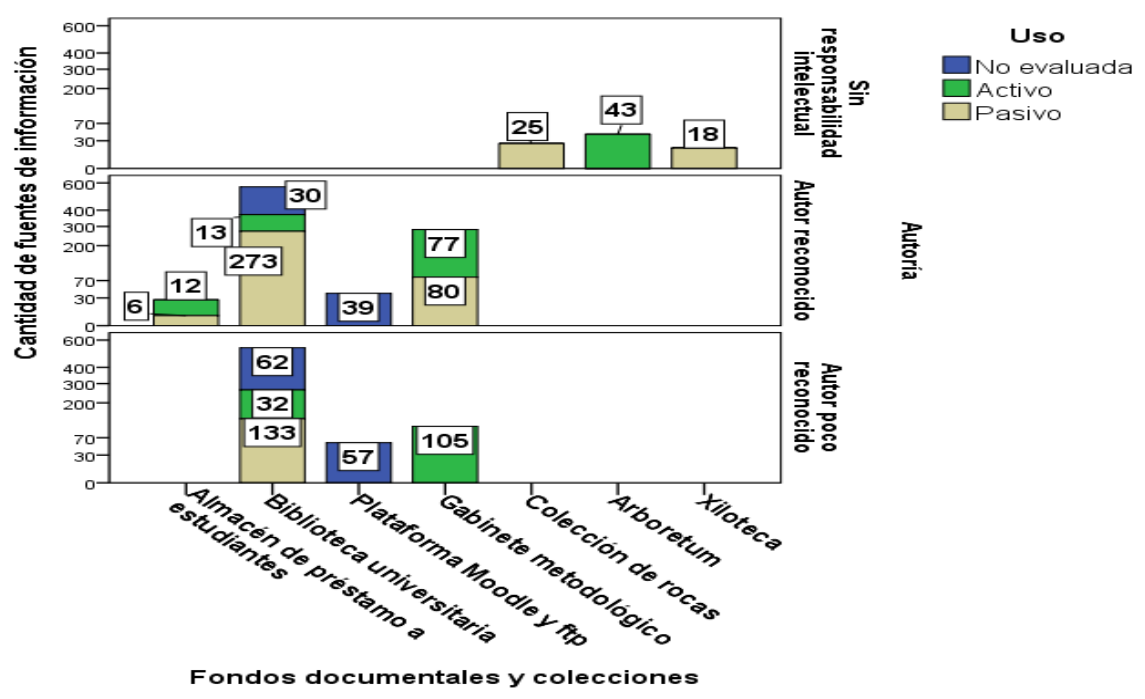

Fig. 6 Uso de las fuentes de información Fuente: Elaboración de los autores

Para los casos específicos de la Xiloteca, Arboretum y la Colección de rocas, solo se usan en las asignaturas Geología, Dendrología, Botánica, Medición y Ecología Forestal.

Para el procesamiento de información científica a desarrollar por los estudiantes en la DPI, se recomienda tener un conocimiento previo del objeto de estudio a partir del trabajo con fuentes de información no documentales y de ellas:

- Identificar la(s) fuente(s) de información y sus características esenciales (consulta de catálogos y manuales establecidos por el Colectivo de Carrera).

- Caracterización físico-geográfica del área relacionada con el objeto de investigación.

- Identificar los autores más reconocidos y las investigaciones que se han desarrollado sobre el tema.

\section{Propuesta de procedimiento}

Como resultado a los datos recogidos, a continuación, se sugieren un conjunto de acciones y operaciones como procedimiento para el procesamiento de información científica en la Disciplina Principal Integradora.

\subsection{Discriminar la información científica}

Determinar las fuentes de información pertinentes en el contexto de la necesidad de información.

\subsubsection{Determinar las fuentes de información documentales adecuadas.}

- Establecer como principal criterio de consulta, los autores más reconocidos en el tema (a través de consulta a especialistas).

- Consultarlas fuentes de información, teniendo en cuenta como orden de relevancia: revista científica (soporte electrónico), libro (electrónico/impreso), Trabajo de Diploma, software, mapa, base de dato, biblioteca digital/virtual, página web, video, videoconferencia, PPT, folleto, programa de TV, otras fuentes de información relacionadas al objeto de investigación. Se excluyen de esta propuesta la guía de estudio, catálogo/manual, diccionario/enciclopedia, pues son fuentes de información secundarias de obligada consulta en la DPI. 
- Evaluar, teniendo en cuenta la descripción física y de contenido de la fuente de información (autor/creador, título, año de publicación/creación, lugar de publicación/creación, índice, resumen), la pertinencia de la información, considerando entre otros elementos, su nivel de actualidad, autenticidad y fiabilidad. Eliminar la duplicidad de información.

- Contrastar las fuentes de información, jerarquizando la relevancia entre ellas, para reducir el número de fuentes de información a aquellas que verdaderamente respondan al tema en cuestión.

\subsubsection{Leer (lectura de estudio) /observar/escuchar}

Se realiza la comprensión e interpretación del contenido de la fuente de información. El estudiante traduce las ideas del autor/creador a sus propios términos. Le otorga significación a lo que percibe.

- Tomar notas: Determinar las palabras clave y/o expresiones relevantes dentro de la fuente de información.

- Identificar, por cada fuente de información, los conceptos fundamentales.

- Establecer relaciones entre dos o más conceptos dentro de una misma fuente de información.

- Establecer comparaciones entre conceptos, criterios y contenidos esenciales, de varias fuentes de información.

- Cuestionar e inferir en torno a los criterios seleccionados del conjunto de fuentes de información.

- Elaborar fichas de contenido de las diferentes fuentes de información consultadas.

\subsection{Sistematizar la información científica}

Representar los conocimientos adquiridos a partir del desarrollo de puntos de vistas propios y el establecimiento de nexos con los conocimientos previos.

\subsubsection{Resumir}

Proceso de reducción cognitiva que contiene información relativa a una fuente original.

- Jerarquizar contenidos.

- Descomponer e integrar (análisis y síntesis).

- Descubrir relaciones subyacentes.

- Este resumen se puede desarrollar en forma de:

- Prosa: Teniendo en cuenta todos los elementos necesarios para una correcta redacción (resumen informativo / indicativo) 
- Gráfico: representación de datos e informaciones a través de figuras.

- Esquema: estructurar de forma lógica el contenido a partir del empleo de términos adecuados.

- Mapa conceptual: "relacionar datos, informaciones y conceptos de una o varias fuentes de información, para descubrir nuevos significados y lograra una mejor aprehensión del contenido" (Van Patten, Chao y Reigeluth, 1986. p.137).

\subsection{Producir información científica}

Construcción y reconstrucción de información según las exigencias del sistema de conocimientos de cada asignatura.

\subsubsection{Establecer conexiones con conocimientos previos.}

- Relacionar la información recuperada con la investigación desarrollada.

- Valorar, emitir juicios y arribar a conclusiones

\subsubsection{Elaborar informes, Trabajos de curso y Trabajo de Diploma.}

- Consultar las guías establecidas en cada asignatura.

- Establecer comparaciones entre los criterios encontrados a partir de semejanzas (puntos de coincidencia) y diferencias (aportes de cada criterio)

- Recolectar los datos a partir del estudio de fuentes de información no documentales.

- Procesar los datos en software (SPSS, STATISTIC, GV SIG, ARGIS, CLIMO PROG, MED TRAB, BIO PRO, CANOCO, STIMATE, SVS)

- Organizar el contenido de forma lógica.

- Determinar las referencias bibliográficas y bibliografía (referenciar los autores más reconocidos en el tema abordado).

\subsubsection{Elaborar ponencias}

- Seleccionar los medios de enseñanza-aprendizaje más adecuados para la presentación de los resultados.

- Seleccionar y explicar los principales resultados.

La valoración del procedimiento se inició con la entrevista a dos especialistas y seis profesores de la DPI, posterior a la revisión del procedimiento por estos. Los especialistas son Licenciados en Español-Literatura, ostentan la Categoría Docente de Profesor Titular y son Doctores en Ciencias Pedagógicas, con una experiencia en la educación superior de 18 y 21 años, respectivamente.

El $100 \%$ de los profesores de la DPI considera al procedimiento necesario, teniendo en cuenta las características de las asignaturas de esta disciplina. Es pertinente enseñarle al estudiante a desarrollar su independencia cognoscitiva a partir de la gran disponibilidad de fuentes de información existentes y donde se convierte en una limitación la precisión para determinar qué s pertinente y qué no lo es. El resultado de las 
entrevistas a especialistas y profesores (Anexo 3) confirma la correspondencia entre las acciones y operaciones propuestas en el procedimiento.

En cuanto al nivel de satisfacción de los profesores respecto a los diferentes fondos y colecciones, son el Gabinete metodológico, la Plataforma Moodle y el ftp, los que cuentan con un mayor reconocimiento por este colectivo, con el $100 \%$ de satisfacción. Los de más baja satisfacción son la Biblioteca universitaria y el almacén de préstamo. Existe correspondencia entre los resultados arrojados por la entrevista y la evaluación realizada de las fuentes de información.

Luego de procesar las entrevistas se realizaron talleres por años académicos, para socializar el procedimiento y enriquecerlo con las sugerencias y criterios de los estudiantes. Entre los principales planteamientos realizados por los estudiantes se encuentran:

- Enfatizar a través de las diferentes asignaturas, en los principales exponentes y sus aportes e incrementar el empleo de software de la carrera, no solo en las asignaturas de la DPI.

- Reconocen la necesidad de fortalecer los hábitos de lectura y la consulta de disímiles fuentes de información.

- Incrementar los documentos en la biblioteca virtual, plataforma moodle y ftp, y dentro de estos, los referidos al procesamiento de información científica (resumen, bibliografías, mapas conceptuales, artículos científicos, lectura)

- Divulgar los principales catálogos y revistas científicas, cubanas y extranjeras, cuyas líneas de investigación se relacionen con la ingeniería Forestal.

- Enfatizar desde el primer año, en el trabajo con las fuentes de información electrónicas, digitales y virtuales.

- Hacer un mayor uso de las plataformas educativas.

- Promover las colecciones de la biblioteca universitaria.

- Mejorar el acceso a las bibliotecas universitarias de otros CES.

Los estudiantes reconocieron la necesidad de aplicar acciones y operaciones que les ayude a optimizar recursos y al mismo tiempo, garantizar la calidad de los resultados alcanzados.

\section{Conclusiones}

Se aporta un procedimiento para el procesamiento de información científica en la DPI, estructurado en tres acciones y un grupo de operaciones, donde se aplican habilidades lógicas y de autoinstrucción, que responden a las limitaciones diagnosticadas.

Las acciones y operaciones propuestas en el procedimiento están dirigidas a: intencionar el trabajo en el uso de las fuentes electrónicas y/o digitales y/o virtuales; desarrollar en los estudiantes habilidades cognitivas para el tratamiento de información científica; hacer un uso óptimo de todas las fuentes de información accesibles y que los estudiantes puedan discriminar informaciones pertinentes de aquellas que no lo son. Se consideran necesarias para el desarrollo del proceso de formación del profesional, teniendo en cuenta las características de la sociedad actual.

Las entrevistas realizadas a especialistas y profesores, así como los talleres desarrollados con los estudiantes a los que está dirigido el procedimiento, corroboran la pertinencia de su aplicación, contribuyendo a la formación de un profesional competente en cuanto al procesamiento de información científica. Se establecen un grupo de relaciones que tributarán a una mayor independencia cognoscitiva del estudiante, reflejada en la realización del trabajo y estudio independientes, en los resultados docentes y en el intercambio estudiante_estudiante, estudiante_profesor. 


\section{Referencias}

Álvarez, C. (1999). Los conocimientos y su clasificación. En: GINORIS, O. (2009). Fundamentos didácticos de la Educación Superior Cubana: Selección de lecturas. La Habana: Editorial Félix Varela.

Cruz, A. (1992). En torno a los conceptos de documento, fuente y recurso de información. Ciencias de la Información. 23 (4), $267-272$.

Cruz, A. y García, V. (1994) Fuentes de Información. Aspectos Teóricos. La Habana: Universidad de La Habana.

Gómez, J. (2004) Neurociencia Cognitiva y Educación. Lambayeque: Fondo Editorial FACHSE.

González, M., Hernández, A. y Viñas, G. (2001) Como ser mejor estudiante. La Habana: CEPES UH.

Kirk, E. (2001). Information and Its Counterfeits: Propaganda, Misinformation and Disinformation. Recuperado de: http://www.library.jhu.edu.

Lancaster, W. y Pinto, M. (2001). Procesamiento de la información científica. S. I: Arco/Libros.

Notario, Á. (1999). Apuntes para un compendio sobre metodología de la investigación científica. Recuperado de: https://dialnet.unirioja.es.

Núñez, I. (2004). La gestión de la información, el conocimiento, la inteligencia y el aprendizaje organizacional desde una perspectiva socio-psicológica. Acimed.12 (3). Recuperado de: http://bvs.sld.cu.

Ruiz, C. (2004). Neurociencia y Educación. En: Gómez, J.(2004).Neurociencia cognitiva y educación. Lambayeque: Fondo Editorial FACHSE.

Tomaél, M. [et. al].(2001). Evaluación de fuentes de información en Internet: Criterios de calidad. Ciencias de la Información, 32 (2), 38.

Van Patten, J., Chao, C. y Reigeluth, C. (1986). A review of strategies for sequencing and synthesizing information. En: Gómez, J. (2004). Neurociencia Cognitiva y Educación.Lambayeque: Fondo Editorial Fachse. 
Procedure for the processing of scientific information in the DPI of the Forest

Engineering programme

Anexo I

Cuestionario a estudiantes sobre el procesamiento de información científica.

Estimado estudiante:

Se está realizando un trabajo de diploma relacionado al procesamiento de información científica desde las diferentes asignaturas de la Disciplina Principal Integradora que usted recibe en este semestre y en el que será de mucha importancia su contribución. Es necesario que la información que nos ofrezca sea fruto de su reflexión. Emplee el tiempo que necesite. Confiamos en su colaboración, la cual le agradecemos de antemano.

\section{Datos generales}

Año Académico:

\section{Cuestionario}

1. Por qué vías obtiene usted las fuentes de información que utiliza en el proceso docente:

Almacén de préstamo a estudiantes de la universidad ( )

A través de la red ( )

Otras ( )

2. Mencione las asignaturas en las que usted ha consultado fuentes de información con mayor sistematicidad. (Menciónelas de mayor a menor uso).

1.

2.

3.

4.

5.

6.

7.

8.

9.

3. ¿Consulta usted documentos en la plataforma Moodle y/o en el FTP?

Sí ( )

4. ¿Qué tipo de fuentes de información consulta con mayor frecuencia?

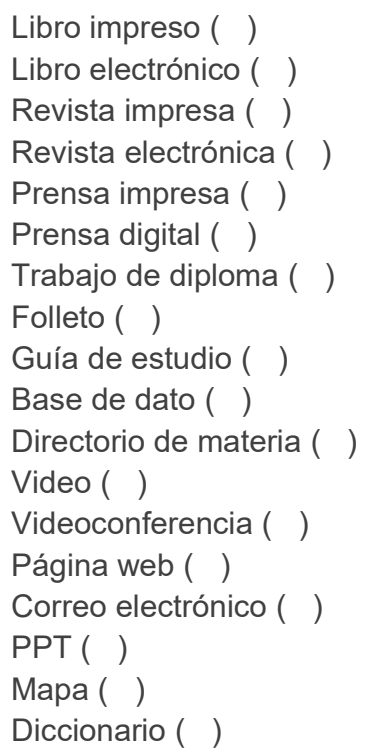


Programa de TV ( )

Software ( )

Biblioteca digital ( )

Biblioteca virtual ( )

Otras ¿Cuáles?

5. ¿Qué nivel de actualización presentan las fuentes de información disponible para usted?

Actualizada ( )

Desactualizada ( )

6. ¿Qué tipo de procesamiento de información realiza ud.?

Lectura de estudio ( )

Resumen ( )

Esquema ( )

Mapa conceptual ( )

Gráfico ( )

Bibliografía ( )

Fichar ( )

Tomar nota ( )

Informe ( )

Trabajo de curso ( )

Ponencia ( )

Otros ¿Cuáles?

7. ¿Qué habilidades emplea usted para el procesamiento de información científica?

Analizar ( )

Sintetizar ( )

Comparar ( )

Abstraer ( )

Caracterizar ( )

Definir ( )

Identificar ( )

Clasificar ( )

Ordenar ( )

Relacionar ( )

Interpretar ( )

Explicar ( )

Observar ( )

Memorizar ( )

Argumentar ( )

Evaluar ( )

Representar ( )

Resumir ( )

Fichar ( )

Anotar (

Otras ¿Cuáles?

8. Aplica usted algún procedimiento para realizar el procesamiento de información científica. De ser afirmativa su respuesta describa este procedimiento.

Sí ( )

No ( ) 
E1010 5 Procedure for the processing of scientific information in the DPI of the Forest Engineering programme

Anexo 2

Entrevista semi-estandarizada a especialistas y profesores de la DPI

9. Datos generales

Especialidad:

Años en la Educación Superior:

Categoría docente:

Categoría científica:

10. ¿Considera usted que el procedimiento propuesto garantiza el adecuado procesamiento de información científica que realizan los estudiantes en la DPI?

Si: $\ldots$

No: ...

¿Por qué?

11. ¿Considera ud. que se debe incluir otro tipo de procesamiento de información científica? ¿Cuál?¿En qué acción?

12. ¿Recomienda usted que se incluyan otras habilidades en el procedimiento?¿Cuáles?¿En qué acción?

13. ¿Considera ud. que el orden propuesto para la consulta de las fuentes de información es adecuado? ¿Tiene usted otra propuesta?

14. ¿Cómo evalúa usted, por fondos y colecciones, las fuentes de información puestas a disposición de los estudiantes?

15. Tiene alguna sugerencia en torno al procedimiento. 
Anexo 3

Resultados de la entrevista a profesores y especialistas

\begin{tabular}{|c|c|c|c|c|}
\hline \multirow[b]{2}{*}{ Pertinencia del procedimiento } & & \multicolumn{3}{|c|}{ Especialidad } \\
\hline & & $\begin{array}{l}\text { Licenciado en Español } \\
\text { Literatura } \\
\end{array}$ & Ingeniero Forestal & Licenciado en Geografía \\
\hline \multirow{2}{*}{$\begin{array}{l}\text { Incorporar otro tipo de procesamiento } \\
\text { de información }\end{array}$} & $\mathrm{Si}$ & 0 & 0 & 0 \\
\hline & No & 2 & 5 & 1 \\
\hline \multirow{2}{*}{ Incorporar otras habilidades } & $\mathrm{Si}$ & 0 & 0 & 0 \\
\hline & No & 2 & 5 & 1 \\
\hline \multirow{3}{*}{$\begin{array}{l}\text { Pertinencia del orden propuesto para la } \\
\text { consulta de fuentes de información }\end{array}$} & & 2 & 5 & 1 \\
\hline & Si & & & \\
\hline & No & 0 & 0 & 0 \\
\hline
\end{tabular}

\section{Dados dos autores}

Ana Luisa Figueredo Figueredo

Licenciada en Bibliotecología y Ciencias de la Información. Máster en Educación Superior. Profesora Auxiliar. Universidad de Granma.

afigueredof@udg.co.cu

Rafael Francisco León Aguilar

Ingeniero Forestal. Universidad de Granma.

afigueredof@udg.co.cu

Mariela María Martínez Roselló

Licenciada en Español-Literatura. Doctora en Ciencias Pedagógicas. Profesora Titular. Universidad de Granma.

afigueredof@udg.co.cu

Recibido - Received: 2017-05-29

Aceptado - Accepted: 2018-12-14

\section{(cc) EY}

This work is licensed under a Creative Commons Attribution 4.0

United States License.

\section{ULIS DDof}

This journal is published by the University Library System of the University of Pittsburgh as part of its

D-Scribe Digital Publishing Program and is cosponsored by the University of Pittsburgh Press. 